\title{
Atributos sensoriais e aceitação de sucos de uva comerciais
}

\author{
Sensory attributes and acceptance of commercial grape juices \\ Pamella Rio Branco PONTES ${ }^{1}$, Savanna Santos SANTIAGO ${ }^{1}$, Tatiana Nogueira SZABO ${ }^{1}$, \\ Luciana Passos TOLEDO ${ }^{1}$, Andréa Pittelli Boiago GOLLÜCKE ${ }^{1 *}$
}

\section{Resumo}

O suco de uva contém compostos fenólicos em quantidades importantes e, portanto, seu consumo é desejável como aporte de substâncias antioxidantes naturais. Os objetivos do estudo foram investigar os atributos sensoriais e avaliar a aceitação de sucos de uva comerciais. Foram analisados três tipos de suco de uva comercializados no Brasil: suco integral, suco concentrado e néctar. Utilizaram-se a Análise Descritiva Quantitativa modificada e o Teste de Aceitação com escala hedônica estruturada de nove pontos, incluindo questões sobre consumo e compra. Os resultados apontam que o suco integral apresentou cor e gosto amargo mais intensos; o suco concentrado (reconstituído) apresentou baixa intensidade em todos os atributos; e o néctar de uva apresentou maior intensidade de gosto doce e sabor característico de uva. O teste de aceitação mostrou que o néctar de uva e o suco integral foram os mais aceitos. A maioria dos consumidores relatou consumir quatro ou mais copos suco de uva por mês. O suco integral recebeu maior intenção de compra e o néctar foi a bebida mais consumida. Concluiu-se que o consumidor aprecia o suco integral da uva, com equilibrada intensidade nos atributos sensoriais investigados, todavia, consume com maior frequência o néctar, bebida à qual são adicionadas água e sacarose.

Palavras-chave: análise sensorial; análise descritiva quantitativa; suco de uva; análise afetiva.

\begin{abstract}
Grape juice contains phenolic compounds in considerable amounts, and it is fit for human consumption since it can increase the intake of natural antioxidants. The objectives of this study were to investigate the sensory attributes and to evaluate the acceptance of commercial grape juices. Three types of commercial grape juices were analyzed: pasteurized juice, concentrated juice, and sugar added pasteurized juice. A modified Quantitative Descriptive Analysis and an Acceptance Test with a structured nine-point hedonic scale were used including questions about consumption and purchase intention. The results indicated that the pasteurized juice showed higher intensity of color and bitterness; the concentrated juice (reconstituted) demonstrated low intensity of all attributes; and the sugar added product showed the highest intensity in sweetness and characteristic grape juice taste. The pasteurized and the sugar added juices were the most accepted products by consumers, who indicated consumption of grape juice of four or more glasses a month. Pasteurized juices received the highest purchase intention scores, and the sugar added juice was the most consumed. Although consumers appreciated pasteurized grape juices with higher intensities of attributes, the sugar added product was the most consumed.
\end{abstract}

Keywords: sensory analysis; grape juice; quantitative descriptive analysis; affective analysis.

\section{Introdução}

As uvas e seus produtos são ricos em compostos fenólicos e vários estudos têm demonstrado que essas substâncias possuem ação anticarcinogênica, antiviral e antioxidante (SGARBIERI; PACHECO, 1999; WILLIAMSON; MANACH, 2005; ARTS; HOLLMAN, 2005; PIMENTEL; FRANCKI; GOLLÜCKE, 2005).

O suco de uva, bebida não-alcoólica, apresenta efeitos como manutenção da função endotelial, diminuição da agregação plaquetária, aumento da capacidade antioxidante e proteção contra oxidação do LDL (O’BYRNE et al., 2002; STEIN et al., 1999). Em 2004, a American Dietetic Association, em seu documento sobre alimentos funcionais, considerou o vinho tinto e o suco de uva como bebidas com evidências "moderadas a fortes" na prevenção da agregação plaquetária em ensaios in vitro, in vivo e em estudos epidemiológicos. Embora as evidências científicas ainda não permitam consenso sobre o consumo desejável, o documento sugere como recomendação preliminar a ingestão diária de 250 a 500 mL (ADA, 2004).

Os diversos tipos de sucos de uva fabricados no Brasil oferecem quantidades relevantes de resveratrol, principalmente para a população que não consome bebida alcoólica derivada da uva (SAUTTER et al., 2005). A substância merece destaque por sua ação antioxidante, anti-inflamatória, antiviral, cardioprotetora e quimiopreventiva de câncer. Está associada também ao retardo do envelhecimento (ACAUAN, 2007). Além das propriedades funcionais, os benefícios nutricionais do suco de uva são evidentes, dada a presença de açúcares e minerais (RIZZON; MIELE, 1995, apud RIZZON; LINK, 2006). Recentemente, Malacrida e Motta (2005 e 2006) investigaram os teores de antocianinas e fenólicos totais em

${ }^{1}$ Curso de Nutrição, Universidade Católica de Santos - UNISANTOS, Av. Conselheiro Nébias, CEP 11015-002, CP 300, Santos - SP, Brasil, E-mail: gollucke@uol.com.br

${ }^{*}$ A quem a correspondência deve ser enviada 
sucos de uva comercializados no Brasil. As autoras encontram teores de fenólicos nos sucos semelhantes aos do vinho tinto, com diferenças significativas entre marcas e tipos de suco. De acordo com Giada e Mancini-Filho (2004) e Bevilaqua (1995), os compostos fenólicos também estão relacionados a propriedades sensoriais como cor, aroma e adstringência dos alimentos.

O consumo de suco de uva no Brasil aumentou significativamente nos últimos anos, passando de 0,15 L per capita, em 1995, para 0,54 L, em 2005 (EMBRAPA, 2006). A produção brasileira de uvas, em 2005, foi de 1,246,071 toneladas, sendo 44,19\% destinadas à produção de vinhos, sucos, destilados e outros derivados. O Estado do Rio Grande do Sul é o principal produtor, os investimentos na viticultura riograndense são importantes por gerarem empregos e renda para as pequenas propriedades (EMBRAPA, 2006). Os tipos de suco de uva comercial vendidos no Brasil são: suco de uva integral em concentração natural; suco de uva concentrado parcialmente desidratado; néctar de uva, obtido pela diluição de suco concentrado em água e com adição de açúcares (BRASIL, 1990, BRASIL, 1997, 2000).

Embora o interesse pelo produto e o consumo do suco de uva tenham aumentado, não foi encontrada na literatura investigação sobre aspectos sensoriais da produção oferecida comercialmente no Brasil. Diante disso e considerando os benefícios nutricionais e funcionais dos sucos de uva, este trabalho teve como objetivo investigar atributos sensoriais desses sucos de uva comerciais e avaliar sua aceitação.

\section{Material e métodos}

Foram avaliados três tipos de suco de uva comercializados no Brasil, representados no Quadro 1.

\subsection{Análise descritiva quantitativa modificada}

O método utilizado foi baseado em Stone e Sidel (2004), com a seleção dos seguintes atributos: gosto amargo, adstringência, gosto doce, cor e sabor característico. Segundo Gollucke, Souza e Tavares (2008), que utilizou o mesmo método em sucos de uva concentrados, $90 \%$ das diferenças sensoriais entre os produtos deveram-se a essas características. A intensidade dos atributos foi avaliada em escala não estruturada de $9 \mathrm{~cm}$, ancorada nos extremos com as palavras nenhum e muito. A compreensão dos atributos e descritores pelos provadores foi verificada e avaliada durante a seleção e treinamento da equipe sensorial.

Quadro 1. Amostras de sucos de uva.

\begin{tabular}{|ll|}
\hline \multicolumn{1}{|c|}{ Tipo } & \multicolumn{1}{c|}{ Ingredientes segundo o rótulo } \\
\hline Néctar de uva & $\begin{array}{l}\text { Água, suco concentrado de uva, açúcar, } \\
\text { aroma idêntico ao natural de uva, } \\
\text { acidulante (ácido cítrico), antioxidante } \\
\text { (ácido ascórbico). }\end{array}$ \\
Suco concentrado de uva & Água potável, suco concentrado de uva \\
- diluído (1:2) conforme & e conservantes (benzoato de sódio e \\
rótulo do fabricante & metabissulfito de sódio). \\
Suco integral de uva & Suco de uva não adoçado, não alcoólico \\
& e não fermentado. \\
\hline
\end{tabular}

Pré-seleção de provadores, desenvolvimento da terminologia descritiva e treinamento da equipe

Foram convidados 60 alunos do $1 .^{\circ}, 2 .^{\circ}$ e $3 .^{\circ}$ anos de um curso universitário na área de alimentos para participar da pesquisa. Os interessados responderam a um questionário com perguntas sobre sua relação sensorial com o produto e situações impeditivas à participação. Foram excluídos os interessados com algum tipo de alergia ou aversão ao suco de uva ou às soluções utilizadas durante o treinamento, sem disponibilidade de tempo ou submetidos a dieta restritiva. Foram aplicados 60 questionários e 26 provadores aptos foram convidados a participar do teste de gostos básicos. O teste consistiu na identificação dos gostos doce, amargo, ácido e salgado. Cada provador recebeu seis soluções (incluindo água e glutamato monossódico), em copos de $30 \mathrm{~mL}$ codificados com números aleatórios de três dígitos, à temperatura ambiente, e indicou o gosto predominante em uma ficha de avaliação. Integraram a equipe aqueles que acertaram todas as seis soluções e também aqueles que acertaram cinco ou quatro, errando somente o gosto salgado e o designado "outro", totalizando 16 provadores. Os participantes assinaram o Termo de Consentimento Livre e Esclarecido aprovado pelo Comitê de Ética da Instituição (Protocolo n. 4096.7.2006).

Durante o treinamento para discriminação dos atributos, as soluções assim como a água para enxaguar a boca foram servidas em temperatura ambiente, também foram oferecidas bolachas de água. Durante as sessões foram definidas, baseado em Behrens e Silva (2000), as concentrações mínima e máxima das soluções de referência que representaram os descritores. Ao final de cada dia de treinamento, os provadores foram avaliados para verificação do uso correto da escala e da correta compreensão de atributos e descritores. Segundo Stone e Sidel (2004), a duração do treinamento é variável dependendo da metodologia utilizada e da experiência dos provadores. Neste trabalho observou-se que reuniões de uma hora por três dias foram adequadas, visto que os provadores eram estudantes da área de alimentos e estavam familiarizados com os atributos e os descritores.

No primeiro dia de treinamento foram discutidos os atributos gosto doce e adstringência; no segundo dia, gosto amargo, cor e sabor característico. No terceiro dia foi feita uma revisão de todos os atributos. Para o treinamento da intensidade dos atributos, cada provador recebeu dois copos, o que continha a substância de referência estava etiquetado com a palavra muito e o que continha água com a palavra nenhum, conforme Quadro 2. Para determinação da intensidade máxima (muito) do atributo sabor característico foram testados os produtos néctar de uva, suco integral e suco concentrado (diluído 1:2).

A eficácia do treinamento foi avaliada após cada sessão de treinamento. Cada provador recebeu uma ficha de avaliação e três copos codificados com amostras de suco ou soluções de referência em diferentes diluições. O treinamento era considerado satisfatório se o provador mostrava-se apto a utilizar a escala e posicionar as amostras ou soluções na ordem correta. Os provadores que apresentaram dificuldades ou erros no uso das escalas receberam orientações. 
Testes

Os testes com as três amostras de sucos de uva comerciais ocorreram no mesmo dia, organizadas segundo o delineamento experimental de blocos completos, balanceados em três repetições. As amostras foram servidas a temperatura ambiente, assim como a água para enxaguar a boca, também foram oferecidas bolachas de água. Na cabine, cada provador recebeu uma amostra codificada por vez, tendo sido observado um intervalo a cada três amostras, totalizando nove. Ao nível de significância de 5\%, a equipe de provadores conseguiu discriminar diferenças entre os cinco atributos estudados $\left(\mathrm{p}_{\text {amostra }}\right)$ e mostrou reprodutibilidade em relação aos atributos $\left(\mathrm{p}_{\text {provador }}\right)$ (Tabela 1$)$.

\subsection{Teste de aceitação}

Noventa e três provadores não treinados, todos acima de 18 anos e predominantemente do sexo feminino (60\%), selecionados entre funcionários, alunos e visitantes da Universidade, considerando-se o interesse e a disponibilidade, participaram do teste de aceitação. Todos os participantes assinaram o Termo de Consentimento Livre e Esclarecido antes da sessão sensorial (Protocolo n. 4096.7.2006).

As três amostras de suco comercial foram avaliadas quanto a sabor, aparência e geral, utilizando escala hedônica estruturada de nove pontos, cujos extremos correspondem a

Quadro 2. Soluções de referência utilizadas no treinamento.

\begin{tabular}{|lll|}
\hline \multicolumn{1}{|c|}{ Atributo } & Nenhum & \multicolumn{1}{c|}{ Muito } \\
\hline Gosto doce & Água & Solução de sacarose a 80 g.L.-1 \\
Adstringência & Água & Solução de ácido tânico a 3 g.L.- \\
Gosto amargo & Água & Solução de cafeína a 1 g.L $L^{-1}$ \\
Cor & Água & Suco de uva concentrado(sem diluição) \\
Sabor característico & Água & Néctar de uva \\
\hline
\end{tabular}

Tabela 1. Nível de significância para avaliação da equipe durante o teste descritivo.

\begin{tabular}{llr}
\hline \multicolumn{1}{c}{ Atributos } & Amostra & Provador \\
\hline Cor & $<0,0001$ & $<0,0001$ \\
Gosto doce & $<0,0001$ & 0,0004 \\
Adstringência & $<0,0001$ & $<0,0012$ \\
Gosto amargo & $<0,0001$ & $<0,0001$ \\
Sabor característico & $<0,0001$ & 0,0366 \\
\hline
\end{tabular}

gostei muitíssimo (9) e desgostei muitíssimo (1). Na mesma ficha foram investigadas a intenção de compra e a frequência de consumo e apresentada uma questão aberta sobre o tipo de suco mais consumido, baseada em Nassu, Lima e Souza-Filho (2001). As amostras foram oferecidas em copos plásticos de 30 $\mathrm{mL}$ à temperatura ambiente. Foram colocadas à disposição dos provadores água para enxaguar a boca e bolachas de água. $\mathrm{Na}$ cabine, cada provador recebeu uma amostra por vez.

\subsection{Análise estatística}

Os resultados dos testes e da avaliação dos provadores foram tratados por análise de variância (ANOVA) e o Teste de Tukey foi usado para verificar diferenças estatísticas entre as amostras, ambos ao nível de 5\%. Foi utilizado o programa estatístico Statistical Analysis Systems, versão 6.08 (SAS, 1992).

\section{Resultados e discussão}

\subsection{Análise descritiva quantitativa modificada}

A Tabela 2 mostra as médias e diferenças significativas ao nível de $5 \%$ entre as amostras de suco integral, concentrado e néctar de uva.

Como se observa na Figura 1, as amostras podem ser assim caracterizadas: o suco integral apresentou cor mais intensa (com valor próximo ao máximo da escala), adstringência e gosto amargo mais intensos, embora ambos em intensidade moderada; o suco concentrado (reconstituído) apresentou cor e gosto doce pouco intensos (este com valor próximo ao mínimo da escala) e as menores intensidades de adstringência e sabor

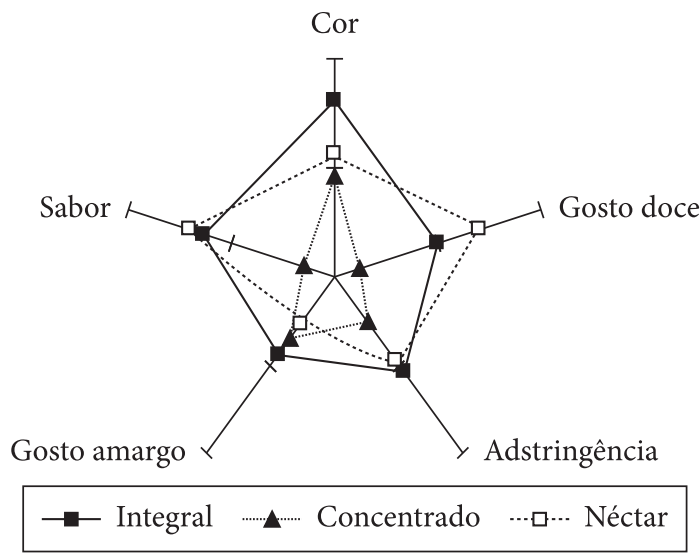

Figura 1. Perfil gráfico das amostras de suco de uva.

Tabela 2. Médias dos atributos sensoriais por amostra.

\begin{tabular}{lccc}
\hline Atributos & Suco integral & Suco concentrado Diluído 1:2 (suco:água) & Néctar de uva \\
\hline Cor & $8,1^{\mathrm{a}}$ & $4,6^{\mathrm{b}}$ & $5,6^{\mathrm{c}}$ \\
Gosto doce & $5,0^{\mathrm{a}}$ & $1,2^{\mathrm{b}}$ & $7,0^{\mathrm{c}}$ \\
Adstringência & $5,4^{\mathrm{a}}$ & $2,5^{\mathrm{b}}$ & $4,7^{\mathrm{a}}$ \\
Gosto amargo & $4,4^{\mathrm{a}}$ & $3,4^{\mathrm{b}}$ & $2,7^{\mathrm{b}}$ \\
Sabor característico & $6,3^{\mathrm{a}}$ & $1,5^{\mathrm{b}}$ & $7,0^{\mathrm{c}}$ \\
\hline
\end{tabular}

Letras diferentes na mesma linha indicam diferença estatística ao nível de $5 \%$. 
característico (ambos com valores próximos ao mínimo); o néctar de uva apresentou a maior intensidade de sabor doce e maior sabor característico de suco de uva.

Della Modesta et al. (2005) desenvolveram perfis sensoriais e avaliaram sucos de maracujá pasteurizados prontos para beber (adicionados de açúcar e água) utilizando a Análise Descritiva Quantitativa (ADQ). Os autores observaram que a sensação de adstringência foi menor em amostras onde o gosto doce apresentou-se mais intenso. No presente trabalho, esta relação não foi observada e o suco concentrado (reconstituído) apresentou baixa intensidade nos dois atributos. Sucos concentrados comercializados a granel e reconstituídos ao teor de sólidos solúveis do suco integral foram avaliados por Gollucke, Souza e Tavares (2008) utilizando a ADQ. Os provadores, neste estudo, atribuíram alta intensidade de gosto doce aos sucos, concordando com o presente estudo, que constatou gosto doce intenso no suco de uva em sua concentração natural e sem adição de sacarose.

\subsection{Teste de aceitação}

Os resultados indicam que o suco integral e néctar de uva foram os mais aceitos pelos provadores, de modo geral e quanto ao sabor, não ocorrendo diferenças estatísticas entre eles. O suco concentrado (reconstituído) foi o menos aceito. Em relação à aparência, o suco integral foi o mais aceito, seguido do néctar (Tabela 3).

Dos 93 provadores que responderam à questão sobre frequência de consumo, $48 \%$ consumiam quatro copos ou mais por mês e $28 \%$ nunca ou pouco consumiam (Figura 2). A questão aberta sobre o tipo de suco consumido apontou que o néctar de uva era o mais consumido (48\%), seguido por outros, classificação que inclui preparados em pó sabor uva (1\% de suco da fruta) e suco à base de extrato de soja. O suco integral

Tabela 3. Médias da aceitação das amostras.

\begin{tabular}{lccc}
\hline Atributos & Néctar de uva & $\begin{array}{c}\text { Suco concentrado } \\
\text { diluído } 1: 2\end{array}$ & Suco integral \\
\hline Geral & $7,3^{\mathrm{a}}$ & $4,2^{\mathrm{b}}$ & $7,3^{\mathrm{a}}$ \\
Sabor & $7,2^{\mathrm{a}}$ & $3,8^{\mathrm{b}}$ & $7,3^{\mathrm{a}}$ \\
Aparência & $7,2^{\mathrm{a}}$ & $5,8^{\mathrm{b}}$ & $7,8^{\mathrm{c}}$ \\
\hline
\end{tabular}

Letras diferentes na mesma linha indicam diferença estatística ao nível de $5 \%$. foi o segundo mais consumido entre os produtos avaliados e o suco concentrado, o terceiro, com apenas $2 \%$ das respostas (Figura 3).

O suco integral apresentou maior intenção de compra (78\% possivelmente comprariam ou certamente comprariam), seguido do néctar (63\%) e do suco concentrado (15\%), dados representados na Figura 4.

Os resultados dos dois testes sensoriais e da avaliação do consumo apontaram o suco integral como o produto com a maior intenção de compra e a mais alta aceitação. Na avaliação dos provadores treinados, este suco apresentou equilíbrio entre os atributos doçura, amargor e adstringência. Foi o suco mais apreciado, junto com o néctar e, no entanto, aparece como o menos consumido. Trata-se de produto mais caro que os demais: o suco natural da uva é embalado em vidro, sem conservantes nem adição de água ou sacarose/edulcorantes.

O néctar de uva teve aceitação equivalente à do suco integral, porém com menor intenção de compra. Obteve a maior intensidade no atributo sabor característico de suco de uva e apresentou desequilíbrio entre os gostos doce e amargo. Revelou-se o produto mais consumido entre os três avaliados. É um suco que apresenta água e sacarose entre seus ingredientes, com preço de venda cerca de 50\% inferior ao do suco integral. O suco concentrado, diluído segundo o rótulo do fabricante, apresentou baixa aceitação, intenção de compra e de consumo. De fato, apresentou baixa intensidade em todos os atributos sensoriais avaliados na Análise Descritiva.

Sucos adoçados parecem ter a preferência do consumidor, conforme demonstrado por Matsuura et al. (2004) no desenvolvimento de um néctar à base de polpa de mamão e suco de maracujá. A adição de açúcar à mistura de frutas diminuiu a acidez e influenciou positivamente a aceitação em até $17 \%$. No entanto, ao elaborar um blend de suco de laranja e cenoura, Branco et al. (2007) verificaram que o teor de sacarose (5 ou $10 \%)$ não influenciou a aceitação dos produtos formulados. No presente estudo, o suco integral contendo os açúcares naturais da fruta apresentou aceitação semelhante ao néctar, que tem adição de sacarose. Constatou-se que os consumidores apreciam os produtos de uva cujos atributos sensoriais são percebidos em alta intensidade e apresentam equilíbrio entre si.

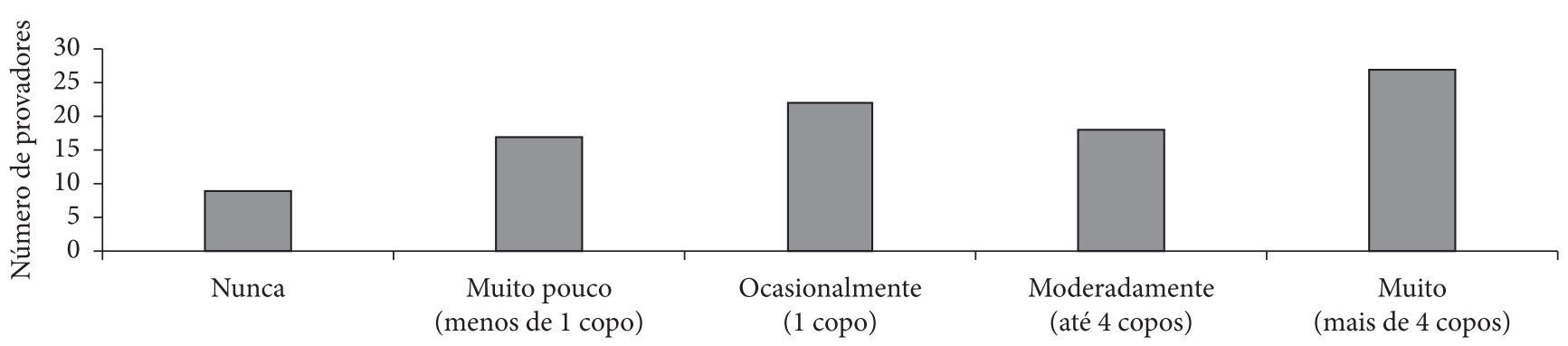

Frequência de consumo ao mês

Figura 2. Frequência de consumo de suco de uva. 


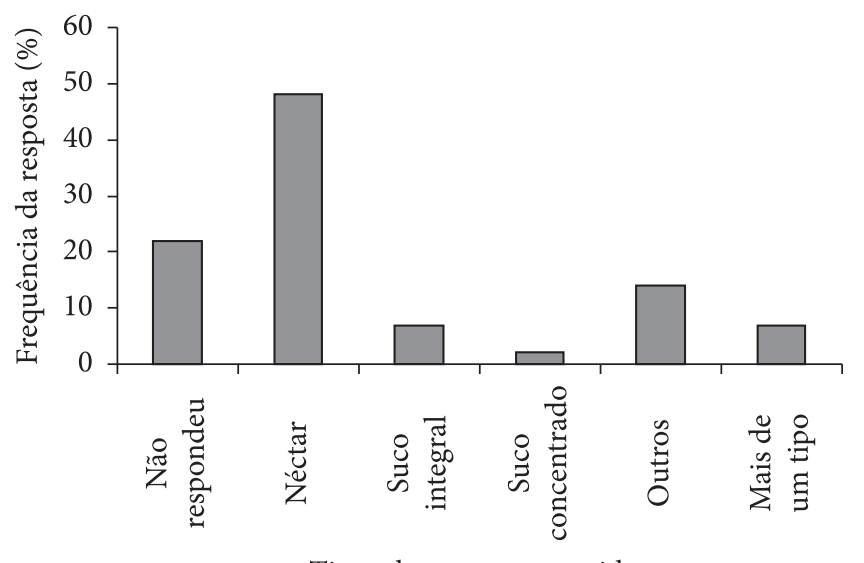

Tipos de sucos consumidos

Figura 3. Frequência de resposta sobre o tipo suco de uva consumido.

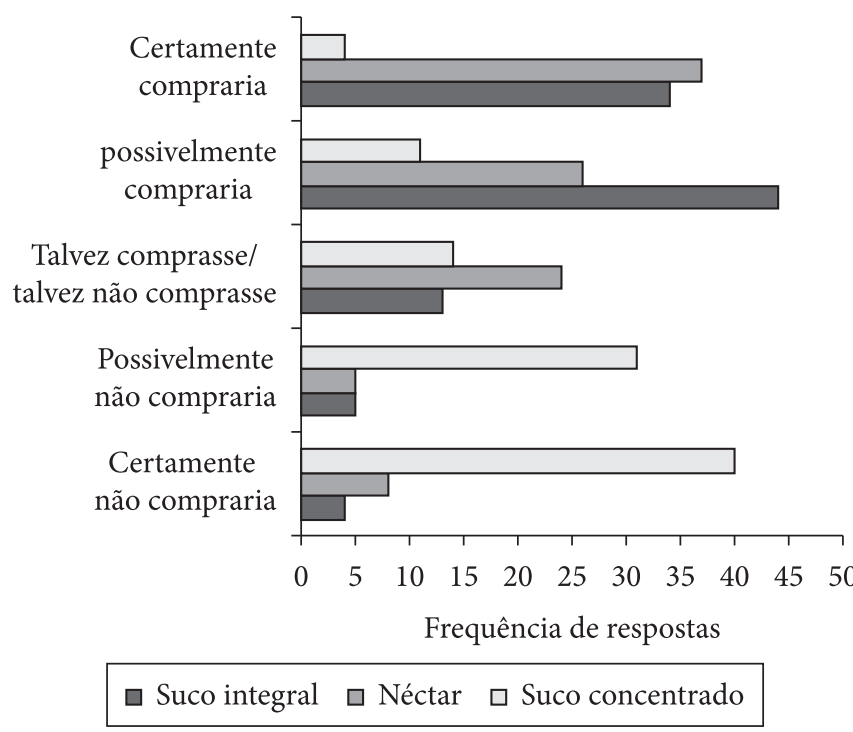

Figura 4. Intenção de compra dos consumidores.

\section{Conclusões}

Esta investigação demonstrou que o suco de uva é um produto apreciado pelos consumidores. Os produtos com maior intensidade de atributos sensoriais e que apresentam equilíbrio entre eles tiveram maior aceitação. O suco integral, produto com alta aceitação é, possivelmente, pouco conhecido pelos consumidores. Os néctares estão mais disponíveis comercialmente, possuem embalagens mais práticas e menor preço, o que provavelmente tem influência no consumo. $\mathrm{O}$ suco de uva em sua concentração original e sem adição de outros ingredientes é uma bebida que agrega benefícios nutricionais e funcionais, aportando antioxidantes à alimentação em quantidades importantes. Com este trabalho, sugere-se à indústria de alimentos a possibilidade de oferecer sucos de uva em sua concentração original, em embalagens práticas, visto que há indícios de mercado consumidor para este produto.

\section{Referências bibliográficas}

ACAUAN, A. P. Supermolécula pode prevenir doenças. Revista da Pontifícia Universidade Católica do Rio Grande do Sul, v. 2, n. 133, p. 6-9, 2007.

AMERICAN DIETETIC ASSOCIATION - ADA. Position of the American Dietetic Association: Functional Foods. Journal of the American Dietetic Association, v. 104, n. 1, p. 814-826, 2004.

ARTS, I. C. W.; HOLLMAN, P. C. H. Polyphenols and disease risk in epidemiologic studies. American Journal of Clinical Nutrition, supl. 81, p. 317-325, 2005.

STONE, H.; SIDEL, J. L. Descriptive Analysis. In: STONE, H.; SIDEL, J. L. Sensory evaluation practices. San Diego: Elsevier Academic Press, 2004. p. 215-235.

BEHRENS, J. H.; SILVA, M. A. A. P. Perfil sensorial de vinhos brancos varietais brasileiros através de análise descritiva quantitativa. Ciência e Tecnologia de Alimentos, v. 20, n. 1, p. 60-67, 2000.

BEVILAQUA, G. A. P. Avaliações físico-químicas durante a maturação de videiras cultivadas no Rio Grande do Sul. Revista Brasileira de Agrociência, v. 1, n. 3, p. 151-156, 1995.

BRANCO, I. G. et al. Avaliação sensorial e estabilidade físico-química de um blend de laranja e cenoura. Revista de Ciência e Tecnologia de Alimentos, v. 27, n. 1, p. 7-12, 2007.

BRASIL. Ministério da Agricultura. Decreto no 99066, 08 de março de 1990: regulamenta a Lei n..$^{\circ}$ 7.678, de 8 de novembro de 1988, que dispõe sobre a produção, circulação e comercialização do vinho e derivados do vinho e da uva. Diário Oficial da União, Brasília, 1990.

BRASIL. Ministério da Saúde. Agência Nacional de Vigilância Sanitária. Regulamenta a Lei no 8.918, 14 de julho de 1994, que dispõe sobre a padronização, a classificação, o registro, a inspeção, a produção e a fiscalização de bebidas. Decreto $n^{\circ} 2314$, de 04 de setembro de 1997. Diário Oficial da União, Brasília, 1997.

BRASIL. Ministério da Saúde. Agência Nacional de Vigilância Sanitária. Decreto $\mathrm{n}^{\circ} 3510,16$ de junho de 2000. Altera dispositivos do Regulamento aprovado pelo Decreto 2.314 de 04 de setembro de 1997, que dispõe sobre a padronização, a classificação, o registro, a inspeção, a produção e a fiscalização de bebidas. Diário Oficial da União, Brasília, 2000.

DELLA MODESTA, R. C. et al. Desenvolvimento do perfil sensorial e avaliação sensorial/instrumental de suco de maracujá. Ciência e Tecnologia de Alimentos, v. 25, n. 2, p. 345-352, 2005.

EMBRAPA. Empresa Brasileira de Pesquisa Agropecuária. Atuação do Brasil no mercado vitivinícula mundial. Disponível em : http://www. cnpuv.embrapa.br/publica/artigos. Acesso em: mar. 2007.

GIADA, M. L. de R.; MANCINI-FILHO, J. Avaliação da atividade antioxidante in vitro de compostos fenólicos de alimentos. Nutrire, v. 28, n. 7, p. 91-107, 2004.

GOLLUCKE, A. P. B.; SOUZA, J. C.; TAVARES, D. Q. Sensory stability of concord and Isabel concentrated grape juices during storage. Journal of Sensory Studies, v. 23, n. 1, p. 340-353, 2008.

MALACRIDA, C. R.; MOTTA, S. Antocianinas em suco de uva: composição e estabilidade. Boletim do Centro de Pesquisa e Processamento de Alimentos, v. 24, n. 1, p. 59-82, 2006.

MALACRIDA, C. R.; MOTTA, S. Compostos fenólicos totais e antocianinas em suco de uva. Ciência e Tecnologia de Alimentos, v. 25, n. 4 , p. 659-664, 2005.

MATSUURA, F. C. A. U. et al. Sensory acceptance of mixed nectar of papaya, passion fruit and acerola. Scientia Agricola, v. 61, n. 6, p. 604-608, 2004. 
NASSU, R. T.; LIMA, J. R.; SOUZA FILHO, M. S. M. Consumers' acceptance of fresh and combined methods processed melon, mango and cashew apple. Revista Brasileira de Fruticultura, v. 23, n. 3 , p. 551-554, 2001.

O'BYRNE, D. J. et al. Comparison of the antioxidant effects of concord grape juice flavonoids and $a$-tocopherol on markers of oxidative stress in healthy adults. American Journal of Clinical Nutrition, n. 76, n. 6, p. 1367-1374, 2002.

PIMENTEL, C. V. M. B.; FRANCKI, V. M.; GOLLÜCKE, A. P. B. Substâncias bioativas em alimentos funcionais. São Paulo: Varela, 2005.

SAS BUSINESS ANALYTICS SOFTWARE. Statistic analisys system. Versão 6.08. Cary, 1992.
SAUTTER, C. K. et al. Determinação de resveratrol em sucos de uva no Brasil. Ciência e Tecnologia de Alimentos, v. 25, n. 3, p. 437-442, 2005.

SGARBIERI, V. C.; PACHECO, M. T. B. Alimentos funcionais fisiológicos. Brazilian Journal of Food Technology, v. 2, n. 1-2, p. 7-19, 1999.

STEIN, J. K. et al. Purple grape juice improves endothelial function and reduces the susceptibility of LDL cholesterol to oxidation in patients with coronary artery disease. Circulation, n. 100, p. 1050-1055, 1999.

WILLIAMSON, G.; MANACH, C. Bioavailability and bioefficacy of polyphenols in humans. II. Review of 93 intervention studies. American Journal of Clinical Nutrition, supl. 81, p. 243-255, 2005. 